\title{
A benchmark database for adsorption bond energies to transition metal surfaces and comparison to selected DFT functionals
}

Jess Wellendorff, ${ }^{1 \$}$ Trent L. Silbaugh, ${ }^{2 \$}$ Delfina Garcia Pintos, ${ }^{1}$ Jens K. Nørskov, ${ }^{1}$ Thomas Bligaard, ${ }^{1}$ Felix Studt, ${ }^{*}$ Charles T. Campbell ${ }^{*}$

(1) SUNCAT Center for Interface Science and Catalysis, SLAC National Accelerator Laboratory, Menlo Park, California 94025, U.S.A. and Department of Chemical Engineering, Stanford University, Stanford, California 94305, U.S.A.

(2) Departments of Chemical Engineering and Chemistry, University of Washington, Box 351700, Seattle, Washington 98195-1700, U.S.A.

\$ These authors contributed equally

*Corresponding authors: studt@slac.stanford.edu, charliec@uw.edu

\begin{abstract}
We present a literature collection of experimental adsorption energies over late transition metal surfaces for systems where we believe the energy measurements are particularly accurate, and the atomic-scale adsorption geometries are particularly well established. We propose that this could become useful for benchmarking theoretical methods for calculating adsorption processes. We compare the experimental results to six commonly used electron density functionals, including some (RPBE, BEEF-vdW) which were specifically developed to treat adsorption processes. The comparison shows that there is ample room for improvements in the theoretical descriptions.
\end{abstract}

\section{KEYWORDS}

Adsorption energies, benchmarking; adsorption; density functional theory; surface science; catalysis; van der Waals; transition metals; single crystal adsorption calorimetry; temperature programmed desorption 


\section{INTRODUCTION}

The application of computational methods such as density functional theory (DFT) calculations to the description of surface-adsorbate bond strength is ever increasing. Often these interactions are used for the evaluation of the performance of solid catalysts in thermal and/or electrochemical reactions. The rapid rise in the number of DFT studies and their use in describing and understanding surface-adsorbate phenomena makes the accuracy of this type of calculation continuously more pertinent to address.[1,2] While higher-level quantum chemical calculations are commonly used in homogeneous catalysis to benchmark results derived from DFT, the evaluation of the reliability of DFT for describing for example adsorption on transition metal surfaces is solely based on comparisons between either different DFT approximations or between DFT and experiments. This is because systematic and higher-level adsorption calculations that are proven to give reliable adsorption energies are still not accessible.

In order to benchmark the theoretical description of heterogeneous catalytic reactions, we need to compare calculated and experimental adsorption energies and reaction barriers of well-defined elementary reaction steps occurring on transition metals and transition metal compounds. Experimental methods to investigate these surface-adsorbate bond strengths are mainly based on equilibrium adsorption studies [3,4], temperature programmed desorption (TPD) [3-5] and single crystal adsorption calorimetry (SCAC) [3,6,7]. Until now, only few experimental adsorption data points have been used to benchmark density functionals [8], and one challenge in obtaining a coherence between theory and experiments for adsorption properties and reaction barriers on surfaces is that the inherent uncertainties are relatively large, both for experiments and theory [8].

In DFT simulations, one can define exactly the atomic-scale adsorption structure or elementary reaction process to be investigated, so for a specific adsorption structure, uncertainties are primarily present as variations between results of different exchange-correlation approximations used to calculate the electronic structure. When comparing the simulations to experiments there is additionally the uncertainty of whether one picked the correct experimental structure to simulate. In the experiments, some uncertainties might arise from the specific experimental setup and calibration techniques and the models used to interpret the experimental data, but a considerable fraction of the uncertainty arises from an uncertainty in exactly which reaction is being observed and how cleanly it occurs. Specifically, in SCAC adsorption experiments one must very carefully consider whether all the adsorbing gas has undergone the dominant reaction expected based on surface 
spectroscopic studies, or if some minor side reactions, surface defects or impurities lead to errors in interpreting the measured heats. Since the theoretical and experimental investigation of adsorption reactions and surface reaction barriers gives very different (conjugated) information and their uncertainties arise in different ways, we can in principle learn a lot from the experiments about how to calibrate the theory, while perhaps also learning from the theory about how to better calibrate the experiments. Such an iterative process has for example taken place with respect to the calculations and measurements of molecular atomization energies in the gas phase [9], and for such properties there is today essentially a perfect correspondence between theory and experiments. Ultimately we should aim to attain the same level of success for describing adsorption phenomena and surface reactions. A prerequisite for such integration of theory and experiment for adsorption properties is that the theoretical and experimental investigations become systematic enough for varying surfaces and adsorbates. In this paper we compile a coherent set of experimental data for adsorption energies of molecules on transition metal surfaces comprised of 39 different reactions measured over 10 different transition metal surfaces, and compare them to results by six common DFT functionals. This data include some of the most recent developments in adsorption energy measurements, but is primarily a compilation and sometimes reinterpretation of existing published experimental data in light of more recent literature with the aim of establishing a set of highly reliable experimental adsorption energies for well-characterized and relatively well-understood systems. The adsorption energies have been calculated here with six exchange-correlation density functionals including LDA [10], PBEsol [11], PW91 [12], PBE [13], RPBE [8], and BEEF-vdW [14] to assess the trends in their relative performance. We divide the adsorption reactions into two benchmark databases, for which the electronic structure calculations exhibit qualitatively different systematic errors. One database contains 25 reactions, where we claim that the adsorbate-surface interactions are predominantly characterized by strong covalent bonds. The second database contains 14 reactions, where we suggest the adsorbate-surface or adsorbateadsorbate bonding has large van der Waals contributions (most or at least 30 $\mathrm{kJ} / \mathrm{mol}$ ) to the total adsorption energies.

To our knowledge, the only previous databases of experimental adsorption energies that have been reported for the purposes of benchmarking DFT have been tabulations that included 11 and 27 energies [8,14], and we have subsequently discovered that several of these energies had rather large calibration errors or were invalid. Those two previous experimental benchmark data sets, while containing many transitions metals, were limited to strongly bound diatomic and atomic species (CO, NO, O [8] and CO, NO, O, H [14]). The 39-energy data set we present 
here refines those lists, while adding weakly and strongly bound hydrocarbon containing up to 18 atoms and iodine-containing species.

\section{METHODS}

Density functional theory calculations were performed using the Quantum ESPRESSO plane-wave code [15] and the Atomic Simulation Environment (ASE) [16]. We used hard PAW pseudopotentials generated using the "atomic" code by Dal Corso [17], except for the case of $\mathrm{Ru}$, where an ultrasoft pseudopotential from the BRV project was employed [18].

Associative and dissociative reactions on transition metal surfaces were modeled using 4-layer metal slabs with at least $10 \AA$ vacuum between the surface species and the bottom of the repeated image of the slab. A dipole correction was applied along the surface normal. The two bottom metal layers were fixed in their bulk geometry (obtained from self-consistently optimizing the bulk lattice constant with the given functional) while the top-two layers and adsorbates were fully relaxed using the BFGS algorithm [19] and a force convergence criterion of $25 \mathrm{meV} / \AA$ along all directions for all relaxing atoms. Clean slab models were relaxed in a similar fashion. The electron temperature was $0.05 \mathrm{eV}$, and spin-polarization was employed for calculations involving $\mathrm{Fe}, \mathrm{Ni}$, and $\mathrm{Co}$. Calculations for isolated molecules were done by centering the molecule in a supercell with at least $8 \AA$ vacuum to any supercell boundary, and then fully relaxing the molecular geometry.

A range of different density functionals was used in this study and for each of them we employed a step-wise procedure for obtaining a well-converged first-principles surface reaction energy. This procedure consists of three steps each using a particular choice of plane-wave cutoff energy and Brillouin zone [20] k-point grid depending on the slab size (see Table 1). The computational load per self-consistent update of the electron density increases significantly from the first to the last set of settings (from Step 1 to Step 3), so applying them successively allowed us to perform relatively cheap geometry optimizations while maintaining reliability of the final reaction energies. Step 1 (S1) is an inexpensive initial relaxation to geometry G1, while Step 2 (S2) takes the atomistic geometry from G1 to its final state G2 in relatively few BFGS steps. Further relaxation at Step 3 (S3) leads in general to no or negligible changes to geometries, so we consider G2 the fully converged atomistic geometry. Step 3 is therefore a high-accuracy fixed-atoms calculation at geometry G2, and it yields the final DFT total energies. 
Table 1: Plane-wave cutoff energy (in Rydbergs) and Gamma-point centered Monkhorst-Pack kpoint grid at each step in the computational procedure. Slab models with three different sizes in the surface plane were considered in this study, and a particular sequence of k-point grids was chosen for each of them. The sequence for relaxing isolated molecules matches the sequences for slab calculations in terms of plane-wave cutoff energy, but samples the Gamma-point only. The cutoff energy for plane-wave expansion of the electron density was always 10 times the wave function cutoff.

\begin{tabular}{|l|c|c|c|}
\hline & $2 \times 2$ slabs & $3 \times 3$ slabs & Molecules \\
\hline Step 1 (S1) & $40 \mathrm{Ry}$ & $40 \mathrm{Ry}$ & $40 \mathrm{Ry}$ \\
& $(4 \times 4 \times 1)$ & $(2 \times 2 \times 1)$ & $(1 \times 1 \times 1)$ \\
Step 2 (S2) & $50 \mathrm{Ry}$ & $50 \mathrm{Ry}$ & $50 \mathrm{Ry}$ \\
& $(6 \times 6 \times 1)$ & $(4 \times 4 \times 1)$ & $(1 \times 1 \times 1)$ \\
\hline \multirow{2}{*}{ Step 3 (S3) } & $60 \mathrm{Ry}$ & $60 \mathrm{Ry}$ & $60 \mathrm{Ry}$ \\
& $(8 \times 8 \times 1)$ & $(6 \times 6 \times 1)$ & $(1 \times 1 \times 1)$ \\
\hline
\end{tabular}

Figure 1 illustrates how the computed surface reaction energies converge towards a well defined value as the procedure outlined above progresses from Step 1 to Step 3. While Step 1 is clearly insufficient for high-accuracy calculations, Step 2 delivers great overall improvements, especially for $(3 \times 3 \times 4)$ slabs with benzene and atomic oxygen adsorbates. It is also apparent from the figure that surface reaction energies on $\mathrm{Ni}$ are slightly underestimated at Step 1, whereas most Pt reaction energies are larger at Step 1 than at Steps 2 and 3.At the final computational step (Step 3) we expect all calculated surface reaction energies to be no more than $0.1 \mathrm{eV}$ different from the perfectly converged (but computationally unattainable) value. This variation is probably within experimental accuracy and has been shown to be comparable to the effect of increasing the number of slab metal layers in the DFT calculations [21]. 


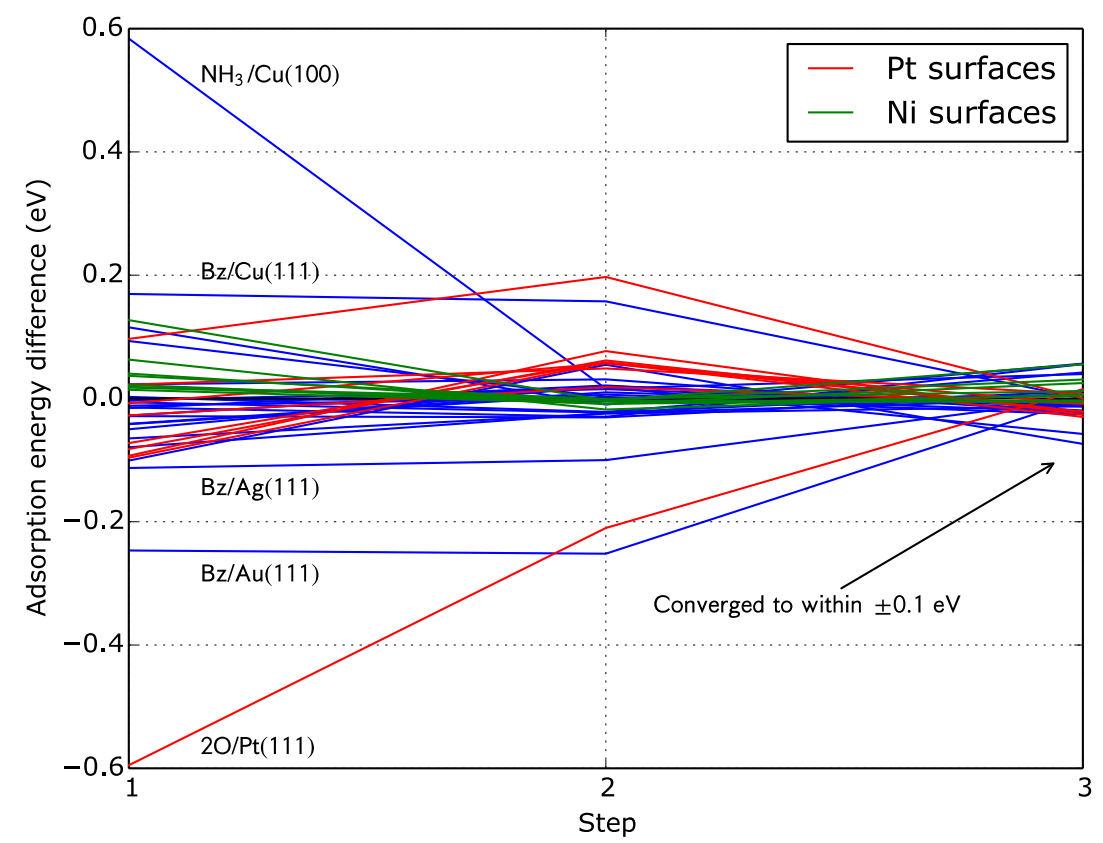

Figure 1: Convergence of a large range of surface adsorption energies with respect to the three steps in the computational procedure. The adsorption energies obtained in each step are compared to those computed with a 70 Ry plane-wave cutoff and even more dense k-point grids than those used at Step 3. Calculations for $t$ and Ni slabs are indicated by red and green colors, respectively. The largest outliers at Step 1 appears to be benzene adsorption on coinage metals, low-coverage $\mathrm{O}$ on Pt(111), and $\mathrm{NH}_{3}$ weakly bound to $\mathrm{Cu}(100)$. This is most likely due to inadequate geometry optimization with the Step 1 settings, which are intended for a fast and inexpensive initial computational step.

The DFT surface reaction energy of the generic process " $A B+M \rightarrow A / M+B$ ", where $A B$ is a gas-phase species, $M$ denotes a metal surface, and $A / M$ and $B$ are adsorbed and desorbed dissociation products, respectively, is defined as

$$
\text { (1) } \Delta \mathrm{E}=\mathrm{E} \text { (products) }-\mathrm{E}(\text { reactants })=\mathrm{E}(\mathrm{A} / \mathrm{M})+\mathrm{E}(\mathrm{B})-\mathrm{E}(\mathrm{AB})-\mathrm{E}(\mathrm{M}) \text {. }
$$

The reaction energy, $\Delta \mathrm{E}$, is a negative quantity for a thermodynamically exothermic process. However, low-temperature experimental surface reaction energies contain vibrational zero-point energy (ZPE) contributions that are not accounted for in the static-lattice DFT reaction energy, Eq. (1). We calculated the ZPE for all considered systems with only the PBE functional, using the harmonic approximation and finite differences $( \pm 0.01 \AA$ displacements along each coordinate of each atom in the adsorbate) to get the normal-mode frequencies. We use these ZPEs to estimate the difference in ZPEs of products minus reactants $(\triangle \mathrm{ZPE})$. (These are listed in Table 3 below, and in Table 4 we subtract these from the experimental reaction energies, resulting in "static-lattice" experimental energies that are directly comparable to DFT.) 
The functionals employed are LDA [10], PBEsol [11], PW91 [12], PBE [13], RPBE [8], and BEEF-vdW [14]. Note that the BEEF-vdW density functional was designed to yield not only accurate energy differences in condensed matter studies, but also estimates of the errors on computed quantities. As illustrated in [2], random sampling of a probability distribution for fluctuations of the BEEF-vdW exchangecorrelation model parameters leads to an ensemble of different predictions of the same quantity. The statistical variance of those predictions defines the squared error estimate on the BEEF-vdW result, $\sigma_{\mathrm{BEE}^{2}}$. This approach to quantitative error estimation in DFT can be viewed as a structured analysis of the sensitivity of DFT results to the choice of exchange-correlation approximation. Furthermore, the energy ensemble is generated post-SCF from 30 non-self-consistent evaluations of the exchange-correlation energy, so obtaining the BEEF-vdW error estimates is a computationally cheap operation.

\section{RESULTS}

ESTABLISHING THE EXPERIMENTAL DATABASE

We start by establishing the experimental database of reaction energies. These were taken entirely from SCAC, TPD, and measurements of equilibrium adsorbate coverages as a function of gas pressure and surface temperature (EAI). Previously established data sets, while containing many transitions metals, were limited to strongly bound diatomic and atomic species (CO, NO, O [8] and CO, NO, O, H [14]).

The database we present here refines the list presented in those earlier publications, while adding weakly and strongly bound hydrocarbon and iodine containing species. In order to ensure that only the most reliable experimental energies are included here, we have omitted any references where invalid assumptions were made in the data analysis (e.g. assuming a pre-exponential factor in TPD that is inconsistent with those determined experimentally for closely-related systems) or where the analysis of raw data that is qualitatively similar to other studies of the same or closely-related systems provided energetics that differ significantly from the consensus in the literature. Studies have also been omitted if surface coverage or the coverage dependence of adsorption energies was not clearly determined. In selecting TPD data, we used only data that had been analyzed by the methods that have been assessed to be the most accurate, as determined in a careful study of different methods [22].

Table 2 contains adsorption enthalpies and energies and surface temperatures that are averages of values reported in the listed references. For a complete list of experimental values used here, see Table S1 in supplemental information. 
It was necessary to recalibrate reported data from several SCAC studies of adsorbates on platinum surfaces and the $\operatorname{Pd}(100)$ facet [7]. It was originally proposed [23], and later corroborated [24], that the reported heats of adsorption for Pt surfaces from the review by Brown et al. [7] require a correction factor of 0.71 due to the use of an incorrect optical reflectivity for Pt (which had been reported incorrectly in the prior literature) during data analysis of the reference heats used for calibration. For a more detailed discussion, see reference [24]. For the $\operatorname{Pd}(100)$ surface, we found that the reported heats of adsorption for CO from SCAC were 26 $\mathrm{kJ} / \mathrm{mol}$ lower than very reliable experimental studies by other methods, which we believe resulted from the same type of error in optical reflectivity (taken from the same prior literature source). From this, we estimated that a correction factor of 1.19 is needed for SCAC values on $\operatorname{Pd}(100)(161 \mathrm{~kJ} / \mathrm{mol} / 135 \mathrm{~kJ} / \mathrm{mol})$ and applied it to the SCAC data from the same group for NO/Pd(100) system. Temperature programmed desorption (TPD) data for benzene on $\mathrm{Cu}(111), \mathrm{Ag}(111)$, and $\mathrm{Au}(111)$ were also reanalyzed using an improved pre-exponential factor of $10^{15.6} \mathrm{~s}^{-1}$ following the report by Campbell and Sellers [25], leading to the improved energies reported in Table 2 . The TPD measurements actually give desorption energies. For these, we only used systems with large enough sticking probabilities that any real activation barrier to adsorption is less than $3 \mathrm{~kJ} / \mathrm{mol}$ (and usually much closer to 0), and corrected them to adsorption energies with the usual 1/2 RT (see supporting information).

For several of the adsorbate systems listed in Table 2, adsorbate-adsorbate interactions lead to the formation of islands with known surface structures. The hexagonal network structure of $\mathrm{D}_{2} \mathrm{O}$ [26] and the $(\sqrt{3} \mathrm{x} \sqrt{3}) \mathrm{R} 30$ of $\left(\mathrm{D}_{2} \mathrm{O}-\mathrm{OD}\right)$ [27] on $\mathrm{Pt}(111)$ are well established in the literature. The structures of adsorbed saturated hydrocarbons (e.g. $\mathrm{C}_{2} \mathrm{H}_{6}, \mathrm{C}_{3} \mathrm{H}_{8}$ ) on $\mathrm{Pt}(111)$ have been extrapolated from the island structure determined for octane on $\mathrm{Pt}(111)$ [28]. Based on this structure, $\mathrm{C}_{n} \mathrm{H}_{n+2}$ chains in a saturated island structure occupy $n+1$ surface sites.

Table 2 shows the database of experimentally measured adsorption energies and reactions on transition metal surfaces. Adsorption energies with multiple references are averages of the values reported (see Table S1 in supplemental information for complete list of adsorption energies from all references).

Table 2. Experimental enthalpies and energies of surface reactions upon gas adsorption, in $\mathrm{kJ} / \mathrm{mol}$ as written.

\begin{tabular}{|c|l|c|l|c|c|c|c|}
$\# \#$ & Surface reaction & $\begin{array}{c}\text { Coverage } \\
(\mathbf{M L})^{\mathbf{1}}\end{array}$ & $\begin{array}{l}\text { Reaction } \\
\text { enthalpy } \\
(\mathbf{k J} / \mathbf{m o l})^{\mathbf{2}}\end{array}$ & $\begin{array}{c}\text { Temp. } \\
(\mathbf{K})\end{array}$ & $\begin{array}{c}\text { Reaction } \\
\text { energy } \\
(\mathbf{k J} / \mathbf{m o l})\end{array}$ & References & Comments \\
\hline $\mathbf{1}$ & $\mathrm{CO}+\mathrm{Ni}(111) \rightarrow \mathrm{CO} / \mathrm{Ni}(111)$ & $1 / 4$ & -122 & 350 & -119 & $5,7,29,30$ & \\
\hline
\end{tabular}




\begin{tabular}{|c|c|c|c|c|c|c|c|}
\hline 2 & $\mathrm{CO}+\mathrm{Pt}(111) \rightarrow \mathrm{CO} / \mathrm{Pt}(111)$ & $1 / 4$ & -120 & 340 & -117 & $\begin{array}{c}7^{*}, 24,31- \\
34\end{array}$ & \\
\hline 3 & $\mathrm{CO}+\mathrm{Pd}(111) \rightarrow \mathrm{CO} / \mathrm{Pd}(111)$ & $1 / 4$ & -143 & 450 & -139 & $35-42$ & \\
\hline 4 & $\mathrm{CO}+\mathrm{Pd}(100) \rightarrow \mathrm{CO} / \mathrm{Pd}(100)$ & $1 / 4$ & -155 & 430 & -151 & $43-45$ & \\
\hline 5 & $\mathrm{CO}+\mathrm{Rh}(111) \rightarrow \mathrm{CO} / \mathrm{Rh}(111)$ & $1 / 4$ & -139 & 500 & -135 & $46-48$ & \\
\hline 6 & $\mathrm{CO}+\operatorname{Ir}(111) \rightarrow \mathrm{CO} / \mathrm{Ir}(111)$ & $1 / 4$ & -158 & 420 & -155 & 49 & \\
\hline 7 & $\mathrm{CO}+\mathrm{Cu}(111) \rightarrow \mathrm{CO} / \mathrm{Cu}(111)$ & $1 / 4$ & -53 & 130 & -52 & 50,51 & \\
\hline 8 & $\mathrm{CO}+\mathrm{Ru}(001) \rightarrow \mathrm{CO} / \mathrm{Ru}(001)$ & $1 / 4$ & -158 & 475 & -154 & 52,53 & \\
\hline 9 & $\mathrm{CO}+\mathrm{Co}(001) \rightarrow \mathrm{CO} / \mathrm{Co}(001)$ & $1 / 4$ & -115 & 370 & -112 & 54 & \\
\hline 10 & $\mathrm{NO}+\mathrm{Ni}(100) \rightarrow \mathrm{N} / \mathrm{Ni}(100)+\mathrm{O} / \mathrm{Ni}(100)$ & $1 / 8$ & -290 & 300 & -288 & 7 & \\
\hline 11 & $\mathrm{NO}+\mathrm{Pt}(111) \rightarrow \mathrm{NO} / \mathrm{Pt}(111)$ & $1 / 4$ & -114 & 300 & -112 & $55^{*}$ & \\
\hline 12 & $\mathrm{NO}+\mathrm{Pd}(111) \rightarrow \mathrm{NO} / \mathrm{Pd}(111)$ & $1 / 4$ & -179 & 520 & -175 & 56 & \\
\hline 13 & $\mathrm{NO}+\mathrm{Pd}(100) \rightarrow \mathrm{NO} / \mathrm{Pd}(100)$ & $1 / 4$ & -161 & 300 & -159 & $7 *$ & \\
\hline 14 & $\mathrm{O}_{2}+\mathrm{Ni}(111) \rightarrow 2 \mathrm{O} / \mathrm{Ni}(111)$ & $1 / 8$ & -480 & 100 & -479 & 7 & \\
\hline 15 & $\mathrm{O}_{2}+\mathrm{Ni}(100) \rightarrow 2 \mathrm{O} / \mathrm{Ni}(100)$ & $1 / 8$ & -530 & 300 & -528 & 7,57 & \\
\hline 16 & $\mathrm{O}_{2}+\mathrm{Pt}(111) \rightarrow 2 \mathrm{O} / \mathrm{Pt}(111)$ & $1 / 18$ & -208 & 515 & -204 & $55^{*}, 58,59$ & \\
\hline 17 & $\mathrm{O}_{2}+\mathrm{Rh}(100) \rightarrow 2 \mathrm{O} / \mathrm{Rh}(100)$ & $1 / 8$ & -358 & 300 & -356 & 7,57 & \\
\hline 18 & $\mathrm{H}_{2}+\mathrm{Pt}(111) \rightarrow 2 \mathrm{H} / \mathrm{Pt}(111)$ & $1 / 8$ & -72 & 300 & -70 & 61,60 & \\
\hline 19 & $\mathrm{H}_{2}+\mathrm{Ni}(111) \rightarrow 2 \mathrm{H} / \mathrm{Ni}(111)$ & $1 / 8$ & -94 & 370 & -91 & 63,64 & \\
\hline 20 & $\mathrm{H}_{2}+\mathrm{Ni}(100) \rightarrow 2 \mathrm{H} / \mathrm{Ni}(100)$ & $1 / 8$ & -94 & 370 & -91 & 63,64 & \\
\hline 21 & $\mathrm{H}_{2}+\mathrm{Rh}(111) \rightarrow 2 \mathrm{H} / \mathrm{Rh}(111)$ & $1 / 8$ & -70 & 325 & -67 & 65 & \\
\hline 22 & $\mathrm{H}_{2}+\mathrm{Pd}(111) \rightarrow 2 \mathrm{H} / \mathrm{Pd}(111)$ & $1 / 8$ & -88 & 370 & -85 & 63,64 & \\
\hline 23 & $\mathrm{I}+\operatorname{Pt}(111) \rightarrow \mathrm{I} / \operatorname{Pt}(111)$ & $1 / 4$ & -230 & 0 & -230 & 66 & $\begin{array}{l}\text { Extrapolated to } 0 \\
\mathrm{~K} .\end{array}$ \\
\hline 24 & $\begin{array}{c}\mathrm{CH}_{2} \mathrm{I}_{2}+\mathrm{Pt}(111) \rightarrow \mathrm{CH} / \mathrm{Pt}(111)+ \\
\mathrm{H} / \mathrm{Pt}(111)+2 \mathrm{I} / \mathrm{Pt}(111)\end{array}$ & $1 / 12$ & -473 & 210 & -471 & 67 & \\
\hline 25 & $\mathrm{CH}_{3} \mathrm{I}+\mathrm{Pt}(111) \rightarrow \mathrm{CH}_{3} / \mathrm{Pt}(111)+\mathrm{I} / \mathrm{Pt}(111)$ & $1 / 25$ & -212 & 320 & -209 & 68 & \\
\hline 26 & $\mathrm{NH}_{3}+\mathrm{Cu}(100) \rightarrow \mathrm{NH}_{3} / \mathrm{Cu}(100)$ & $1 / 4$ & -57 & 235 & -55 & 69 & \\
\hline 27 & $\mathrm{CH}_{3} \mathrm{I}+\mathrm{Pt}(111) \rightarrow \mathrm{CH}_{3} \mathrm{I} / \mathrm{Pt}(111)$ & $1 / 4$ & -84.5 & 100 & -83.7 & 70 & \\
\hline 28 & $\mathrm{CH}_{3} \mathrm{OH}+\mathrm{Pt}(111) \rightarrow \mathrm{CH}_{3} \mathrm{OH} / \mathrm{Pt}(111)$ & $1 / 4$ & -56 & 100 & -55 & 71 & \\
\hline 29 & $\mathrm{CH}_{4}+\mathrm{Pt}(111) \rightarrow \mathrm{CH}_{4} / \mathrm{Pt}(111)$ & $1 / 2$ & -15 & 63 & -14.5 & 72 & Methane \\
\hline 30 & $\mathrm{C}_{2} \mathrm{H}_{6}+\mathrm{Pt}(111) \rightarrow \mathrm{C}_{2} \mathrm{H}_{6} / \mathrm{Pt}(111)$ & $1 / 3$ & -28.5 & 106 & -27.6 & 72 & Ethane \\
\hline 31 & $\mathrm{C}_{3} \mathrm{H}_{8}+\mathrm{Pt}(111) \rightarrow \mathrm{C}_{3} \mathrm{H}_{8} / \mathrm{Pt}(111)$ & $1 / 4$ & -41.3 & 139 & -40.1 & 72 & Propane \\
\hline 32 & $\mathrm{C}_{4} \mathrm{H}_{10}+\mathrm{Pt}(111) \rightarrow \mathrm{C}_{4} \mathrm{H}_{10} / \mathrm{Pt}(111)$ & $1 / 5$ & -50.8 & 171 & -49.4 & 72 & n-butane \\
\hline 33 & $\mathrm{C}_{6} \mathrm{H}_{6}+\mathrm{Pt}(111) \rightarrow \mathrm{C}_{6} \mathrm{H}_{6} / \mathrm{Pt}(111)$ & $1 / 9$ & -164 & 300 & -162 & 31,73 & Benzene \\
\hline 34 & $\mathrm{C}_{6} \mathrm{H}_{6}+\mathrm{Cu}(111) \rightarrow \mathrm{C}_{6} \mathrm{H}_{6} / \mathrm{Cu}(111)$ & $1 / 9$ & -68 & 225 & -66 & 74,75 & Benzene \\
\hline 35 & $\mathrm{C}_{6} \mathrm{H}_{6}+\mathrm{Ag}(111) \rightarrow \mathrm{C}_{6} \mathrm{H}_{6} / \mathrm{Ag}(111)$ & $1 / 9$ & -63 & 210 & -61 & 76 & Benzene \\
\hline 36 & $\mathrm{C}_{6} \mathrm{H}_{6}+\mathrm{Au}(111) \rightarrow \mathrm{C}_{6} \mathrm{H}_{6} / \mathrm{Au}(111)$ & $1 / 9$ & -72 & 230 & -70 & 77 & Benzene \\
\hline 37 & $\mathrm{C}_{6} \mathrm{H}_{10}+\operatorname{Pt}(111) \rightarrow \mathrm{C}_{6} \mathrm{H}_{10} / \mathrm{Pt}(111)$ & $1 / 9$ & -122 & 100 & -121 & 62 & Cyclohexene \\
\hline 38 & $\mathrm{D}_{2} \mathrm{O}+\mathrm{Pt}(111) \rightarrow \mathrm{D}_{2} \mathrm{O} / \mathrm{Pt}(111)$ & $\sim 2 / 3$ & -51.3 & 120 & -50.3 & 26 & $\begin{array}{l}\text { Hexagonal } \\
\text { network, big } \\
\text { islands }\end{array}$ \\
\hline 39 & $\begin{array}{l}\mathrm{D}_{2} \mathrm{O}+1 / 3 \mathrm{O} / \mathrm{Pt}(111) \rightarrow \\
2 / 3\left(\mathrm{D}_{2} \mathrm{O} \cdots \mathrm{OD}\right) / \mathrm{Pt}(111)\end{array}$ & $1 / 2$ & -57.4 & 150 & -56.2 & 27 & \\
\hline
\end{tabular}


See more extensive table in Supporting Information for details on individual values that went into these averages.

* This entry was corrected from the original value reported as described in the text.

${ }^{1}$ These coverages are defined here as the number of reacted gas molecules in the reaction as written per metal surface atom. For the dissociative chemisorption of a diatomic molecule, a coverage of " $1 / 8$ " thus means that there will be 1 atomic adsorbate per 4 metal surface atoms.

${ }^{2}$ These are the enthalpy changes for the reaction per mole of reacted gas as written, integrated from zero coverage of the reacted gas up to its listed coverage. The enthalpy changes are converted to changes in internal energy by adding the small correction of $+\mathrm{RT}(=+2.5 \mathrm{~kJ} / \mathrm{mol}$ at $300 \mathrm{~K})$.

${ }^{3}$ The temperatures reported here represent the average temperature at which experiments were performed, or for TPD experiments, the temperature that yields the highest desorption rate, unless otherwise noted. For systems where multiple references are given, the temperature is taken as the average from all experiments cited.

\section{PERFORMANCE OF DFT FUNCTIONALS}

Table 3 shows the calculated reaction energies without ZPE corrections for a variety of density functionals which are commonly used for the description of bulk materials and surface reactions. The reaction ZPE corrections are also shown in Table $3(\triangle \mathrm{ZPE}$, the difference in ZPE between products minus reactant calculated with PBE). In order to establish a useful benchmark set of experimentally defined adsorption systems, we subtract the calculated $\triangle \mathrm{ZPE}$ contributions from the experimental reaction energies from Table 2. This value of $\Delta \mathrm{E}_{\exp }-\Delta \mathrm{ZPE}$ is shown in Tables $4 \mathrm{a}$ and $4 \mathrm{~b}$ for the benchmark set of reactions. This choice of modifying the experiment with the calculated $\triangle \mathrm{ZPE}$ makes it possible to directly compare the theoretically obtained reaction energies to the benchmark data. 
Table 3. Theoretical surface reaction energies, in $\mathrm{kJ} / \mathrm{mol}$ as written ${ }^{1,2)}$.

\begin{tabular}{|c|c|c|c|c|c|c|c|c|c|c|}
\hline$\#$ & Surface reaction & $\begin{array}{l}\text { Adsorbate } \\
\text { coverage as } \\
\text { written }^{3)}\end{array}$ & $\begin{array}{c}\Delta \mathrm{ZPE} \\
\text { via } \\
\mathrm{PBE}\end{array}$ & $\mathrm{LDA}^{9)}$ & PBEsol $^{9)}$ & PW919) & $\mathrm{PBE}^{9)}$ & $\mathrm{RPBE}^{9)}$ & BEEF-vdW ${ }^{9}$ & $\begin{array}{c}\sigma_{\mathrm{BEE}} \\
5)\end{array}$ \\
\hline 1 & $\mathrm{CO}+\mathrm{Ni}(111) \rightarrow \mathrm{CO} / \mathrm{Ni}(111)$ & $1 / 4$ & 4.7 & -268 & -221 & -177 & -175 & -140 & -146 & 17 \\
\hline 2 & $\mathrm{CO}+\operatorname{Pt}(111) \rightarrow \mathrm{CO} / \mathrm{Pt}(111)$ & $1 / 4$ & 7.5 & -213 & -184 & -156 & -156 & -136 & -133 & 21 \\
\hline 3 & $\mathrm{CO}+\mathrm{Pd}(111) \rightarrow \mathrm{CO} / \mathrm{Pd}(111)$ & $1 / 4$ & 5.5 & -268 & -229 & -188 & -175 & -153 & -156 & 20 \\
\hline 4 & $\mathrm{CO}+\mathrm{Pd}(100) \rightarrow \mathrm{CO} / \mathrm{Pd}(100)$ & $1 / 4$ & 6.1 & -255 & -217 & -181 & -164 & -154 & -150 & 14 \\
\hline 5 & $\mathrm{CO}+\mathrm{Rh}(111) \rightarrow \mathrm{CO} / \mathrm{Rh}(111)$ & $1 / 4$ & 7.4 & -244 & -214 & -185 & -184 & -163 & -162 & 11 \\
\hline 6 & $\mathrm{CO}+\operatorname{Ir}(111) \rightarrow \mathrm{CO} / \operatorname{Ir}(111)$ & $1 / 4$ & 8.6 & -244 & -216 & -190 & -189 & -169 & -170 & 14 \\
\hline 7 & $\mathrm{CO}+\mathrm{Cu}(111) \rightarrow \mathrm{CO} / \mathrm{Cu}(111)$ & $1 / 4$ & 4.9 & -132 & -103 & -75 & -73 & -56 & -55 & 14 \\
\hline 8 & $\mathrm{CO}+\mathrm{Ru}(001) \rightarrow \mathrm{CO} / \mathrm{Ru}(001)$ & $1 / 4$ & 7.1 & -240 & -212 & -184 & -182 & -162 & -162 & 13 \\
\hline 9 & $\mathrm{CO}+\mathrm{Co}(001) \rightarrow \mathrm{CO} / \mathrm{Co}(001)$ & $1 / 4$ & 6.8 & -234 & -193 & -162 & -148 & -137 & -138 & 14 \\
\hline 10 & $\begin{array}{l}\mathrm{NO}+\mathrm{Ni}(100) \rightarrow \mathrm{N} / \mathrm{Ni}(100)+ \\
\mathrm{O} / \mathrm{Ni}(100)\end{array}$ & $\begin{array}{c}1 / 4 \\
(\text { separated })^{4)}\end{array}$ & 11.4 & -578 & -488 & -429 & -401 & -375 & -370 & 44 \\
\hline 11 & $\mathrm{NO}+\operatorname{Pt}(111) \rightarrow \mathrm{NO} / \mathrm{Pt}(111)$ & $1 / 4$ & 6.6 & -276 & -219 & -177 & -166 & -148 & -149 & 17 \\
\hline 12 & $\mathrm{NO}+\mathrm{Pd}(111) \rightarrow \mathrm{NO} / \mathrm{Pd}(111)$ & $1 / 4$ & 6.8 & -315 & -258 & -217 & -202 & -188 & -186 & 15 \\
\hline 13 & $\mathrm{NO}+\mathrm{Pd}(100) \rightarrow \mathrm{NO} / \mathrm{Pd}(100)$ & $1 / 4$ & 4.1 & -311 & -250 & -204 & -185 & -172 & -174 & 16 \\
\hline 14 & $\mathrm{O}_{2}+\mathrm{Ni}(111) \rightarrow 2 \mathrm{O} / \mathrm{Ni}(111)$ & $1 / 8$ & 6.2 & -566 & -488 & -456 & -444 & -393 & -418 & 43 \\
\hline 15 & $\mathrm{O}_{2}+\mathrm{Ni}(100) \rightarrow 2 \mathrm{O} / \mathrm{Ni}(100)$ & $1 / 8$ & 2.5 & -641 & -555 & -510 & -465 & -444 & -471 & 38 \\
\hline 16 & $\mathrm{O}_{2}+\mathrm{Pt}(111) \rightarrow 2 \mathrm{O} / \mathrm{Pt}(111)$ & $1 / 18$ & 4.4 & -313 & -241 & -218 & -206 & -159 & -190 & 43 \\
\hline 17 & $\mathrm{O}_{2}+\mathrm{Rh}(100) \rightarrow 2 \mathrm{O} / \mathrm{Rh}(100)$ & $1 / 8$ & -0.6 & -552 & -470 & -421 & -368 & -355 & -387 & 38 \\
\hline 18 & $\mathrm{H}_{2}+\mathrm{Pt}(111) \rightarrow 2 \mathrm{H} / \mathrm{Pt}(111)$ & $1 / 8$ & 1.7 & -160 & -129 & -94 & -79 & -66 & -48 & 21 \\
\hline 19 & $\mathrm{H}_{2}+\mathrm{Ni}(111) \rightarrow 2 \mathrm{H} / \mathrm{Ni}(111)$ & $1 / 8$ & 8.5 & -184 & -144 & -107 & -104 & -79 & -64 & 17 \\
\hline 20 & $\mathrm{H}_{2}+\mathrm{Ni}(100) \rightarrow 2 \mathrm{H} / \mathrm{Ni}(100)$ & $1 / 8$ & -4.4 & -182 & -145 & -102 & -85 & -68 & -56 & 17 \\
\hline 21 & $\mathrm{H}_{2}+\mathrm{Rh}(111) \rightarrow 2 \mathrm{H} / \mathrm{Rh}(111)$ & $1 / 8$ & 4.8 & -180 & -148 & -112 & -112 & -84 & -67 & 15 \\
\hline 22 & $\mathrm{H}_{2}+\mathrm{Pd}(111) \rightarrow 2 \mathrm{H} / \mathrm{Pd}(111)$ & $1 / 8$ & 5.2 & -184 & -151 & -116 & -91 & -88 & -67 & 18 \\
\hline 23 & $\mathrm{I}_{2}+\operatorname{Pt}(111) \rightarrow 2 \mathrm{I} / \mathrm{Pt}(111)^{6}$ & $1 / 8$ & 1.4 & -376 & -329 & -269 & -251 & -229 & -269 & 38 \\
\hline 24 & $\begin{array}{l}\mathrm{CH}_{2} \mathrm{I}_{2}+\mathrm{Pt}(111) \rightarrow \mathrm{CH} / \mathrm{Pt}(111)+ \\
\mathrm{H} / \mathrm{Pt}(111)+2 \mathrm{I} / \mathrm{Pt}(111)\end{array}$ & $\begin{array}{c}1 / 4 \\
(\text { separated })^{4)}\end{array}$ & -15.6 & -521 & -453 & -363 & -333 & -286 & -340 & 66 \\
\hline 25 & $\begin{array}{l}\mathrm{CH}_{3} \mathrm{I}+\mathrm{Pt}(111) \rightarrow \mathrm{CH}_{3} / \mathrm{Pt}(111)+ \\
\mathrm{I} / \mathrm{Pt}(111)\end{array}$ & $\begin{array}{c}1 / 4 \\
(\text { separated })^{4)}\end{array}$ & 0.0 & -240 & -200 & -159 & -140 & -114 & -164 & 22 \\
\hline 26 & $\mathrm{NH}_{3}+\mathrm{Cu}(100) \rightarrow \mathrm{NH}_{3} / \mathrm{Cu}(100)$ & $1 / 4$ & 5.5 & -87 & -60 & -42 & -39 & -36 & -41 & 19 \\
\hline 27 & $\mathrm{CH}_{3} \mathrm{I}+\mathrm{Pt}(111) \rightarrow \mathrm{CH}_{3} \mathrm{I} / \mathrm{Pt}(111)$ & $1 / 4$ & -0.02 & -76 & -49 & -26 & -23 & -16 & -36 & 19 \\
\hline 28 & $\begin{array}{l}\mathrm{CH}_{3} \mathrm{OH}+\mathrm{Pt}(111) \rightarrow \\
\mathrm{CH}_{3} \mathrm{OH} / \mathrm{Pt}(111)\end{array}$ & $1 / 4$ & 0.02 & -70 & -37 & -20 & -17 & -16 & -31 & 20 \\
\hline 29 & $\mathrm{CH}_{4}+\mathrm{Pt}(111) \rightarrow \mathrm{CH}_{4} / \mathrm{Pt}(111)$ & $1 / 4$ & -0.6 & -21 & -5 & -3 & -2 & -1 & -15 & 13 \\
\hline 30 & $\mathrm{C}_{2} \mathrm{H}_{6}+\mathrm{Pt}(111) \rightarrow \mathrm{C}_{2} \mathrm{H}_{6} / \mathrm{Pt}(111)$ & $1 / 9$ & -0.5 & -43 & -15 & -5 & -3 & -3 & -21 & 18 \\
\hline 31 & $\mathrm{C}_{3} \mathrm{H}_{8}+\mathrm{Pt}(111) \rightarrow \mathrm{C}_{3} \mathrm{H}_{8} / \mathrm{Pt}(111)$ & $1 / 9$ & -0.8 & -63 & -23 & -6 & -5 & -4 & -29 & 23 \\
\hline 32 & $\mathrm{C}_{4} \mathrm{H}_{10}+\operatorname{Pt}(111) \rightarrow \mathrm{C}_{4} \mathrm{H}_{10} / \mathrm{Pt}(111)$ & $1 / 9$ & -1.5 & -81 & -33 & -9 & -7 & -6 & -38 & 28 \\
\hline 33 & $\mathrm{C}_{6} \mathrm{H}_{6}+\mathrm{Pt}(111) \rightarrow \mathrm{C}_{6} \mathrm{H}_{6} / \mathrm{Pt}(111)$ & $1 / 9$ & -0.4 & -265 & -193 & -90 & -89 & -10 & -76 & 52 \\
\hline 34 & $\begin{array}{l}\mathrm{C}_{6} \mathrm{H}_{6}+\mathrm{Cu}(111) \rightarrow \\
\mathrm{C}_{6} \mathrm{H}_{6} / \mathrm{Cu}(111)\end{array}$ & $1 / 9$ & -0.4 & -77 & -35 & -27 & -23 & -20 & -82 & 79 \\
\hline 35 & $\begin{array}{l}\mathrm{C}_{6} \mathrm{H}_{6}+\mathrm{Ag}(111) \rightarrow \\
\mathrm{C}_{6} \mathrm{H}_{6} / \mathrm{Ag}(111)\end{array}$ & $1 / 9$ & -0.1 & -61 & -14 & -6 & 5 & 10 & -21 & 39 \\
\hline
\end{tabular}




\begin{tabular}{|c|l|c|c|c|c|c|c|c|c|c|}
36 & $\begin{array}{l}\mathrm{C}_{6} \mathrm{H}_{6}+\mathrm{Au}(111) \rightarrow \\
\mathrm{C}_{6} \mathrm{H}_{6} / \mathrm{Au}(111)\end{array}$ & $1 / 9$ & -0.3 & -39 & 4 & 17 & 20 & 22 & -18 & 30 \\
\hline 37 & $\mathrm{C}_{6} \mathrm{H}_{10}+\mathrm{Pt}(111) \rightarrow \mathrm{C}_{6} \mathrm{H}_{10} / \mathrm{Pt}(111)$ & $1 / 9$ & 2.2 & -177 & -125 & -74 & -70 & -23 & -85 & 26 \\
\hline 38 & $\mathrm{H}_{2} \mathrm{O}+\mathrm{Pt}(111) \rightarrow \mathrm{H}_{2} \mathrm{O} / \mathrm{Pt}(111)$ & $1 / 4^{7)}$ & 5.0 & -63 & -37 & -21 & -19 & -5 & -21 & 15 \\
\hline & $\begin{array}{l}\mathrm{H}_{2} \mathrm{O}+1 / 3 \mathrm{O} / \mathrm{Pt}(111) \rightarrow \\
39\end{array}$ & $1 / 2^{8}\left(\mathrm{H}_{2} \mathrm{O} \cdots \mathrm{OH}\right) / \mathrm{Pt}(111)$ & 9.8 & -122 & -85 & -62 & -59 & -30 & -52 & 24 \\
\hline
\end{tabular}

1) These are the energy changes for the reaction per mole of reacted gas as written, integrated from zero coverage of the reacted gas up to its listed coverage.

2) see supporting information for side and top view of calculated structures.

${ }^{3)}$ Coverages here are defined as in Table 2, i.e., the number of reacted gas molecules in the reaction as written per metal surface atom, except as otherwise noted.

${ }^{4)}$ In these reactions where two or more different adsorbates are produced, each adsorbate was calculated separately at the stated coverage of that adsorbate alone, and then the energies of these separated adlayers were properly summed. Thus, the local coverages here are different from those reported in Table 2, but chosen to get the computations done within reasonable timeframes.

5) The BEEF-vdW error estimate for each surface reaction is indicated in the last column, labeled " $\sigma_{\mathrm{BEE}}$ ". See text for explanation.

${ }^{6}$ ) Calculated values for this reaction are referenced to molecular $\mathrm{I}_{2}$ gas, instead of an iodine radical as used in Table 2. Using $\mathrm{I}_{2}$ (gas) as the reactant together with the experimental zero-Kelvin dissociation energy of $I_{2}$ gas $(148.5 \mathrm{~kJ} / \mathrm{mol}$,) [78] changes the experimental value from Table 2 to $\Delta \mathrm{E}_{\text {expimental }}=2 *-230 \mathrm{~kJ} / \mathrm{mol}+(148.5 \mathrm{~kJ} / \mathrm{mol})=-312 \mathrm{~kJ} / \mathrm{mol}$, for direct comparison to these numbers.

7) The coverage of reaction 36 is different from that reported in Table 2.

8) This is the same structure as in Fig. 1c of reference [79].

9) DFT calculated reaction energies, not including ZPE corrections. 
Table 4a. Errors in theoretical surface reaction energies for reactions leading to strongly covalently adsorbed species, i.e. chemisorbed systems (in $\mathrm{kJ} / \mathrm{mol}$ as written).

\begin{tabular}{|c|c|c|c|c|c|c|c|c|c|c|}
\hline \multirow[b]{2}{*}{$\#$} & \multirow[b]{2}{*}{ Surface reaction } & \multirow[b]{2}{*}{$\begin{array}{l}\Delta \mathrm{E}_{\text {exp }}- \\
\Delta \mathrm{ZPE}^{2)}\end{array}$} & \multicolumn{7}{|c|}{ Surface reaction energy errors $(\mathrm{kJ} / \mathrm{mol})($ negative $=$ overbinding $)$} & \multirow[b]{2}{*}{ Weight ${ }^{1}$} \\
\hline & & & LDA & PBEsol & PW91 & PBE & RPBE & BEEF-vdW & $\sigma_{\mathrm{BEE}}$ & \\
\hline 1 & $\mathrm{CO}+\mathrm{Ni}(111) \rightarrow \mathrm{CO} / \mathrm{Ni}(111)$ & -124 & -144 & -97 & -53 & -51 & -16 & -22 & 17 & 1 \\
\hline 2 & $\mathrm{CO}+\mathrm{Pt}(111) \rightarrow \mathrm{CO} / \mathrm{Pt}(111)$ & -124 & -88 & -60 & -32 & -32 & -12 & -8 & 21 & 1 \\
\hline 3 & $\mathrm{CO}+\mathrm{Pd}(111) \rightarrow \mathrm{CO} / \mathrm{Pd}(111)$ & -144 & -124 & -84 & -44 & -30 & -8 & -12 & 20 & 1 \\
\hline 4 & $\mathrm{CO}+\mathrm{Pd}(100) \rightarrow \mathrm{CO} / \mathrm{Pd}(100)$ & -157 & -98 & -60 & -24 & -7 & 3 & 7 & 14 & 1 \\
\hline 5 & $\mathrm{CO}+\mathrm{Rh}(111) \rightarrow \mathrm{CO} / \mathrm{Rh}(111)$ & -142 & -102 & -72 & -43 & -42 & -21 & -20 & 11 & 1 \\
\hline 6 & $\mathrm{CO}+\operatorname{Ir}(111) \rightarrow \mathrm{CO} / \mathrm{Ir}(111)$ & -164 & -80 & -52 & -26 & -25 & -5 & -6 & 14 & 1 \\
\hline 7 & $\mathrm{CO}+\mathrm{Cu}(111) \rightarrow \mathrm{CO} / \mathrm{Cu}(111)$ & -57 & -75 & -46 & -18 & -16 & 1 & 2 & 14 & 1 \\
\hline 8 & $\mathrm{CO}+\mathrm{Ru}(001) \rightarrow \mathrm{CO} / \mathrm{Ru}(001)$ & -161 & -79 & -51 & -23 & -21 & -1 & -1 & 13 & 1 \\
\hline 9 & $\mathrm{CO}+\mathrm{Co}(001) \rightarrow \mathrm{CO} / \mathrm{Co}(001)$ & -119 & -115 & -74 & -43 & -29 & -18 & -19 & 14 & 1 \\
\hline 10 & $\begin{array}{l}\mathrm{NO}+\mathrm{Ni}(100) \rightarrow \mathrm{N} / \mathrm{Ni}(100)+ \\
\mathrm{O} / \mathrm{Ni}(100)\end{array}$ & -299 & -279 & -189 & -130 & -102 & -76 & -71 & 44 & 0.5 \\
\hline 11 & $\mathrm{NO}+\mathrm{Pt}(111) \rightarrow \mathrm{NO} / \mathrm{Pt}(111)$ & -119 & -157 & -100 & -58 & -47 & -29 & -30 & 17 & 1 \\
\hline 12 & $\mathrm{NO}+\mathrm{Pd}(111) \rightarrow \mathrm{NO} / \mathrm{Pd}(111)$ & -182 & -133 & -76 & -35 & -20 & -6 & -4 & 15 & 1 \\
\hline 13 & $\mathrm{NO}+\mathrm{Pd}(100) \rightarrow \mathrm{NO} / \mathrm{Pd}(100)$ & -163 & -148 & -87 & -41 & -22 & -9 & -11 & 16 & 1 \\
\hline 14 & $\mathrm{O}_{2}+\mathrm{Ni}(111) \rightarrow 2 \mathrm{O} / \mathrm{Ni}(111)$ & -485 & -81 & -3 & 29 & 41 & 92 & 67 & 43 & 0.5 \\
\hline 15 & $\mathrm{O}_{2}+\mathrm{Ni}(100) \rightarrow 2 \mathrm{O} / \mathrm{Ni}(100)$ & -530 & -110 & -24 & 20 & 66 & 86 & 60 & 38 & 0.5 \\
\hline 16 & $\mathrm{O}_{2}+\mathrm{Pt}(111) \rightarrow 2 \mathrm{O} / \mathrm{Pt}(111)$ & -208 & -105 & -33 & -10 & 2 & 49 & 18 & 43 & 0.5 \\
\hline 17 & $\mathrm{O}_{2}+\mathrm{Rh}(100) \rightarrow 2 \mathrm{O} / \mathrm{Rh}(100)$ & -355 & -197 & -115 & -66 & -13 & 0 & -32 & 38 & 0.5 \\
\hline 18 & $\mathrm{H}_{2}+\mathrm{Pt}(111) \rightarrow 2 \mathrm{H} / \mathrm{Pt}(111)$ & -72 & -88 & -57 & -22 & -7 & 6 & 24 & 21 & 0.5 \\
\hline 19 & $\mathrm{H}_{2}+\mathrm{Ni}(111) \rightarrow 2 \mathrm{H} / \mathrm{Ni}(111)$ & -100 & -84 & -44 & -8 & -4 & 20 & 36 & 17 & 0.5 \\
\hline 20 & $\mathrm{H}_{2}+\mathrm{Ni}(100) \rightarrow 2 \mathrm{H} / \mathrm{Ni}(100)$ & -87 & -95 & -58 & -15 & 2 & 19 & 31 & 17 & 0.5 \\
\hline 21 & $\mathrm{H}_{2}+\mathrm{Rh}(111) \rightarrow 2 \mathrm{H} / \mathrm{Rh}(111)$ & -72 & -108 & -76 & -40 & -40 & -12 & 5 & 15 & 0.5 \\
\hline 22 & $\mathrm{H}_{2}+\mathrm{Pd}(111) \rightarrow 2 \mathrm{H} / \mathrm{Pd}(111)$ & -90 & -94 & -61 & -26 & -1 & 2 & 23 & 18 & 0.5 \\
\hline 23 & $\mathrm{I}_{2}+\mathrm{Pt}(111) \rightarrow 2 \mathrm{I} / \mathrm{Pt}(111)$ & -313 & -63 & -16 & 44 & 62 & 84 & 44 & 38 & 0.5 \\
\hline 24 & $\begin{array}{l}\mathrm{CH}_{2} \mathrm{I}_{2}+\mathrm{Pt}(111) \rightarrow \mathrm{CH} / \mathrm{Pt}(111)+ \\
\mathrm{H} / \mathrm{Pt}(111)+2 \mathrm{I} / \mathrm{Pt}(111)\end{array}$ & -455 & -66 & 2 & 92 & 122 & 169 & 115 & 66 & 0.25 \\
\hline 25 & $\begin{array}{l}\mathrm{CH}_{3} \mathrm{I}+\mathrm{Pt}(111) \rightarrow \mathrm{CH}_{3} / \mathrm{Pt}(111)+ \\
\mathrm{I} / \mathrm{Pt}(111)\end{array}$ & -209 & -31 & 9 & 50 & 69 & 95 & 45 & 22 & 0.5 \\
\hline \multicolumn{11}{|c|}{ Error averages for chemisorption reactions (kJ/mol) (Reactions 1-25) } \\
\hline \multicolumn{11}{|c|}{\begin{tabular}{|l|l|l|l|l|l|} 
Surface reaction energy errors: & & & & &
\end{tabular}} \\
\hline & MSE & & -109.8 & -61.0 & -20.8 & -5.8 & 16.6 & 9.6 & & \\
\hline & MAE & & 109.8 & 61.9 & 39.7 & 35.0 & 33.7 & 28.5 & 24.3 & \\
\hline & RMSE & & 119.9 & 73.3 & 47.5 & 46.2 & 53.7 & 38.8 & & \\
\hline \multicolumn{11}{|c|}{$\begin{array}{l}\text { Reaction energy errors per } \\
\text { adsorbed fragment: }\end{array}$} \\
\hline & Weighted MSE ${ }^{1)}$ & & -81.1 & -47.7 & -20.1 & -11.0 & 4.2 & 1.2 & & \\
\hline & Weighted MAE $^{1)}$ & & 81.1 & 48.1 & 27.7 & 23.1 & 17.7 & 16.0 & 15.4 & \\
\hline & Weighted RMSE ${ }^{\text {1) }}$ & & 91.4 & 57.4 & 32.2 & 27.8 & 23.9 & 18.8 & & \\
\hline
\end{tabular}


1) The weighted errors have been chosen so that the error expresses the mean DFT errors on a "per adsorbed fragment" basis thus reflecting the DFT energy errors on a per surface-adsorbate bond basis. 2) Experimentally determined reaction energies (from column 6 in Table 2) where the theoretically calculated $\triangle \mathrm{ZPE}$ from PBE (column 4 in Table 3 ) is subtracted. 
Table 4b. Errors in theoretical surface reaction energies for reactions where the adsorbate bonding is estimated to have large contributions from dispersion interactions, i.e., primarily involving weakly interacting closed shell adsorbates (in $\mathrm{kJ} / \mathrm{mol}$ as written).

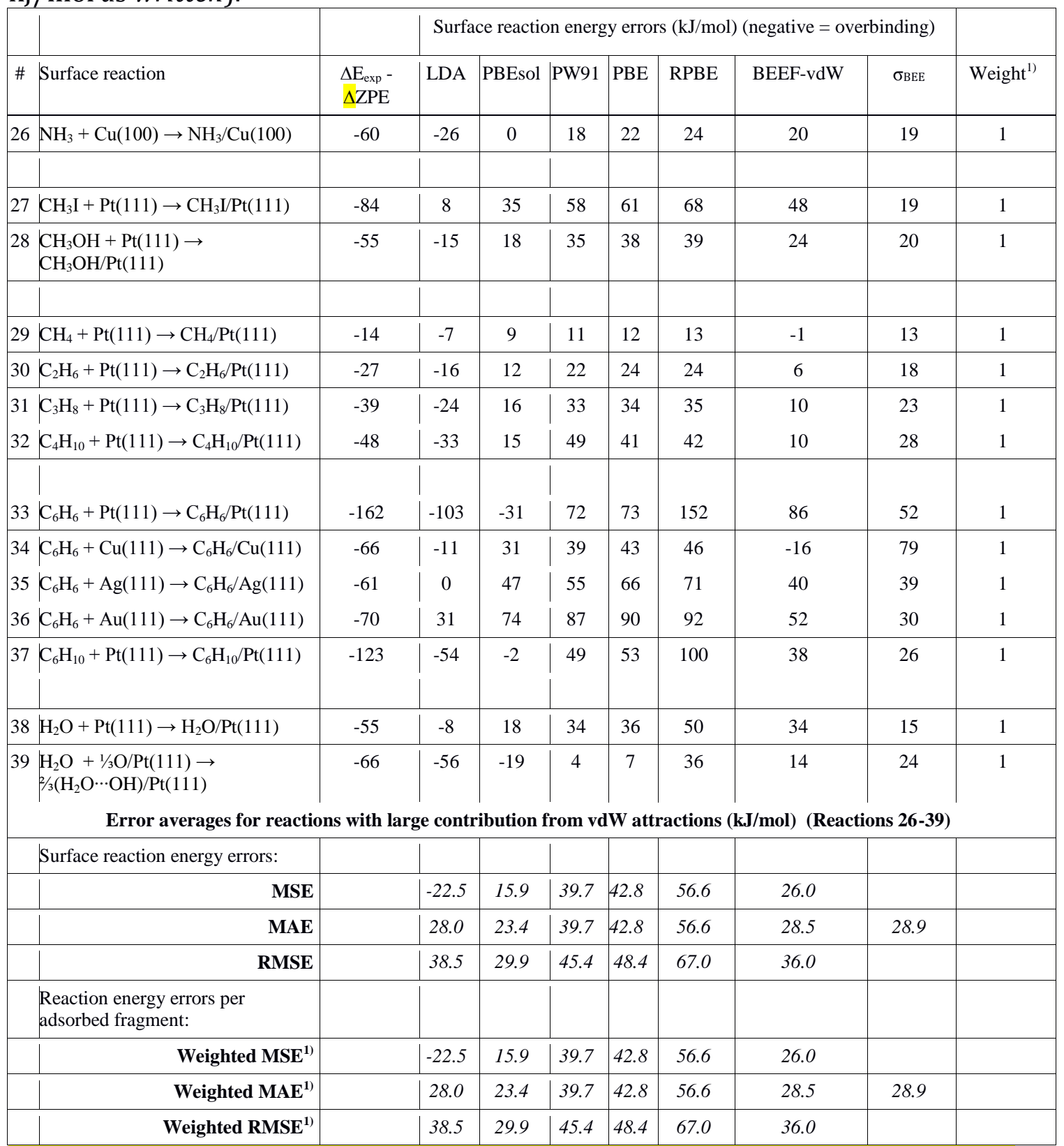

1) The weighted errors have been chosen so that the error expresses the mean DFT errors on a "per adsorbed fragment" basis thus reflecting the DFT energy errors on a per surface-adsorbate bond basis. 2) Experimentally determined reaction energies (from column 6 in Table 2) where the theoretically calculated $\triangle \mathrm{ZPE}$ from PBE (column 4 in Table 3 ) is subtracted. 
A closer look at Table 2 shows that the reactions considered are rather different in nature. Reactions 1-25 are molecular and dissociative adsorption processes where strong surface-adsorbate bonds are formed. Reactions 26-39 on the other hand involve a large contribution from closed shell adsorbates so that van der Waals interactions dominate bonding or contribute to a large extend. It is quite revealing to observe the performance of the various functionals for these adsorption processes in more detail. Thus, comparison of the overall performance of the various density functionals with respect to the ZPE-corrected experimental reaction energies is shown in Tables $4 \mathrm{a}$ for chemisorbed systems (reactions 1-25) and 4b for systems where vdW interactions make a large contribution to the total adsorption energy (reactions 26-39).

Average surface reaction energy errors are computed in terms of the mean signed error (MSE), the mean absolute error (MAE), and the root-mean squared error (RMSE). These statistics are shown in Table $4 \mathrm{a}$ and $\mathrm{b}$. Tables $4 \mathrm{a}$ and $4 \mathrm{~b}$ also contain weighted average error quantities, where the weights (last column in both tables) have been chosen such that the weighted average expresses the mean DFT errors on a "per adsorbed fragment" basis. These averages are intended to reflect the DFT energy error on a "per surface-adsorbate bond" basis. These weighted error averages are also illustrated in Figure 2. For comparison, the average reaction energy $\left(\Delta \mathrm{E}_{\exp }-\Delta \mathrm{ZPE}\right)$ for all 25 chemisorption reactions in Table $4 \mathrm{a}$ is -197.2 $\mathrm{kJ} / \mathrm{mol}$, but only $-66.4 \mathrm{~kJ} / \mathrm{mol}$ for the 14 reactions with large van der Waals contribution in Table $4 \mathrm{~b}$. 

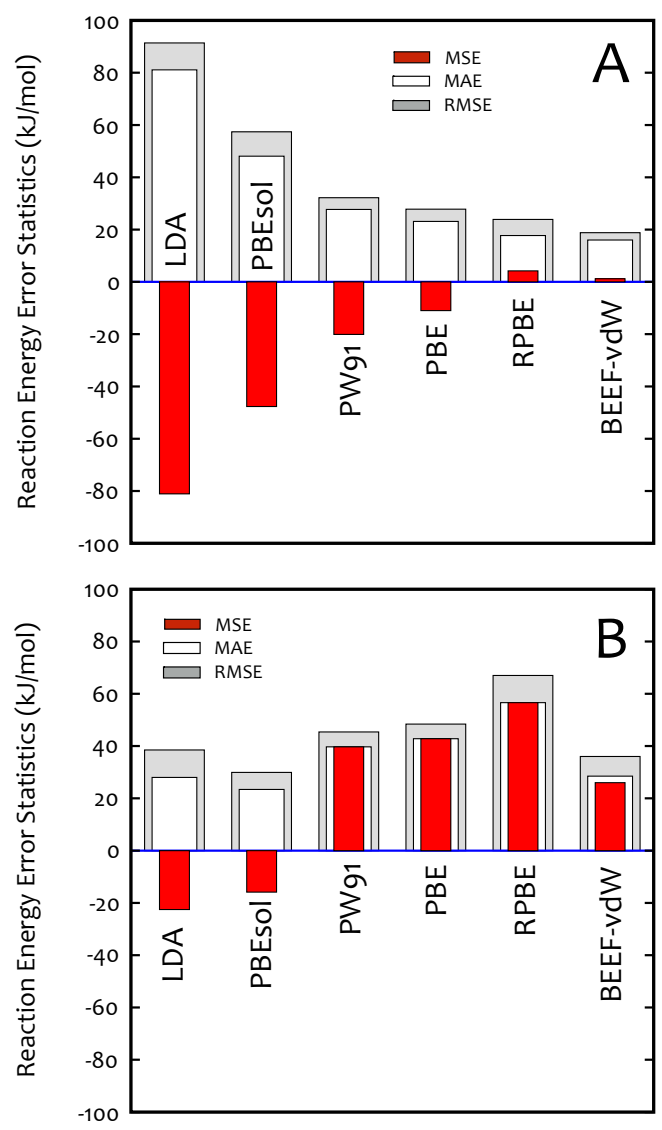

Figure 2: Comparison of the accuracy of the different density functionals relative to the experimental data of Table 2 for two different datasets: (A) Purely chemisorbed systems where van der Waals ( $v d W)$ interactions contribute little to the adsorption energy (reactions 1-25), and (B) Systems where vdW interactions are thought to contribute a large amount to the adsorption energy (due to large contributions from closed-shell adsorbates, reactions 26- 39). For each dataset, the bars illustrate the weighted mean standard error (MSE, red bars, which includes the sign of the error in the average, the weighted mean absolute error, (MAE, white bars, which uses absolute values without signs) and the weighted root mean squared error (RMSE, grey bars).

Inspection of Table 4a, where the errors for chemisorption processes (reactions 125) are shown, revels that PW91 overbinds most chemisorbed systems significantly. PBE still overbinds, but performs somewhat better, whereas both RPBE and BEEF$v d W$ that have been fitted to describe surface adsorbate bonds perform best.

When considering energies that have large contributions from van der Waals interactions (Table $4 \mathrm{~b}$, reactions 26-39), it is noted that the strong overbinding of the PW91 functional leads also to a better description of the systems with large vdW contributions compared to RPBE, which does not overbind in general. RPBE performs quite well for all chemisorbed systems for which it was optimized, but fails badly for species with large vdW contributions. BEEF-vdW, a functional that is 
similar to RPBE but includes the Langreth-Lundqvist non-local correlation [80], shows reasonable performance for both systems with and without large vdW contributions (see Tables $4 \mathrm{a}$ and $4 \mathrm{~b}$ and Figure 2).

In general, however, it can be noticed that the MAE and RMSE errors of chemisorbed systems (Table 4a and Figure 2a) of the various functionals decrease from LDA $>$ PBEsol $>$ PW91 $>$ PBE $>$ RPBE $>$ BEEF-vdW, with the BEEF-vdW functional showing errors of $16.0 \mathrm{~kJ} / \mathrm{mol}$ (MAE) and $18.8 \mathrm{~kJ} / \mathrm{mol}$ (RMSE). For systems with large contributions from van der Waals interactions, RPBE performs worst, followed by PW91 and PBE, with BEEF-vdW showing the smallest errors (28.5 for MAE and 36.0 for RMSE). Overall, it seems that reactions involving NO, $\mathrm{O}_{2}$, and benzene are particularly poorly described. For NO, all functionals predict too strong binding, while for $\mathrm{O}_{2}$ too weak dissociative chemisorption is predicted. Benzene chemisorption for the reactive $\mathrm{Pt}(111)$ surface is particularly poor for all functionals except PBEsol. LDA overbinds rather strongly while all other functionals predict a too weak chemisorption energy ranging from $+72 \mathrm{~kJ} / \mathrm{mol}$ (PW91) to $+152 \mathrm{~kJ} / \mathrm{mol}$ (RPBE). While part of this discrepancy is due to missing description of dispersion forces, we note that even BEEF-vdW underestimates the binding by $86 \mathrm{~kJ} / \mathrm{mol}$.

One does not yet know exactly how to classify surface reactions into these two classes, since there is no well-defined way to know what contribution to the adsorption energy really comes from van der Waals interactions. Consider, for example, reactions \#23-24 where two adsorbed iodine adatoms are products (as written in the calculated reactions of Tables 3-4). Since iodine atoms are large, the contribution of van der Waals interactions to the heat of adsorption of two of these may be large. So it is somewhat unclear to which group these two reactions belong.

Finally, we note that the BEEF-vdW error estimates, second last column of Figures $4 \mathrm{a}$ and $4 \mathrm{~b}$, reproduce the BEEF-vdW prediction errors reasonably well. Slightly more than half of the observed errors are numerically larger than the corresponding error estimate, and 4 of the 39 observed errors are more than twice as large as the error estimate. This is slightly more than expected for a normal distribution, and it indicates that the error estimate is slightly smaller than it should be. This most likely occurs since the benchmark adsorption set presented here is slightly more diverse than what the functional was fitted to. However, the error estimate is certainly the right order of magnitude, and as illustrated in Ref. 2, the BEEF-vdW error estimates may therefore be very useful in computational surface science studies, and are essentially available free of cost once each self-consistent DFT calculation is done.

It is interesting to further discuss the classes of reactions that show larger errors. The error in chemisorption energies could be due to a poor description of surfaceadsorbate bonds, the adsorbate itself in the gas-phase or both. It has been found that rather simple gas-phase molecules such as $\mathrm{O}_{2}$ and $\mathrm{CO}_{2}$ are poorly described by some functionals [24,81-83]. The problem seems to be located in the description of double and triple bonds of gas-phase molecules while fully hydrogenated derivatives of 
these molecules are usually described rather well. Table 5 shows the error for 5 theoretical gas-phase reactions $(1 \mathrm{~g}-5 \mathrm{~g})$ involving $\mathrm{NO}, \mathrm{O}_{2}, \mathrm{CO}, \mathrm{N}_{2}$, and benzene. 
Table 5. Errors in theoretical gas-phase reaction energies (in kJ/mol).

\begin{tabular}{|c|l|c|c|c|c|c|c|c|}
\hline \multicolumn{2}{|c|}{} & \multicolumn{5}{|c|}{ Reaction energy errors (kJ/mol as written) } \\
\hline $1 \mathrm{~g}$ & Gas-phase reaction & $\begin{array}{c}\text { Benchmark } \\
(\mathrm{kJ} / \mathrm{mol})^{1)}\end{array}$ & LDA & PBEsol & PW91 & PBE & RPBE & BEEF-vdW \\
$2 \mathrm{~g}$ & $\mathrm{NO}+5 / 2 \mathrm{H}_{2} \rightarrow \mathrm{CH}_{4}+\mathrm{NH}_{2}+\mathrm{H}_{2} \mathrm{O}$ & -272 & -105 & -63 & -30 & -27 & 9 & 37 \\
\hline $3 \mathrm{~g}$ & $\mathrm{O}_{2}+2 \mathrm{H}_{2} \rightarrow 2 \mathrm{H}_{2} \mathrm{O}$ & -529 & -25 & 21 & 41 & 46 & 73 & 82 \\
\hline $4 \mathrm{~g}$ & $\mathrm{~N}_{2}+3 \mathrm{H}_{2} \rightarrow 2 \mathrm{NH}_{3}$ & -163 & -125 & -78 & -41 & -37 & 2 & 29 \\
\hline $5 \mathrm{~g}$ & $\mathrm{C}_{6} \mathrm{H}_{6}+3 \mathrm{H}_{2} \rightarrow \mathrm{C}_{6} \mathrm{H}_{12}$ & -285 & -78 & -28 & 18 & 20 & 65 & 74 \\
\hline
\end{tabular}

1) Computed from benchmark CCSD(T) atomization energies from [84], except for reaction $5 g$, where benchmark zero-Kelvin formation energies from the G3/99 test set were used [85].

It is interesting to note that the functionals investigated here show quite different performance for the various gas-phase reactions. LDA predicts too large reactions energies with Reaction $3 \mathrm{~g}$ involving $\mathrm{O}_{2}$ being closest to the experimental value. PBEsol is similar to LDA, but somewhat improved. PW91, PBE, RPBE, and BEEFvdW all perform rather differently for the various reactions. PW91 and PBE are accurate for $\mathrm{NO}$ and benzene, RPBE for $\mathrm{CO}$ and $\mathrm{N}_{2}$ and BEEF-vdW underestimates all reactions energies by at least $30 \mathrm{~kJ} / \mathrm{mol}$. For some reaction energies, e.g. Reaction $3 \mathrm{~g}$ involving $\mathrm{O}_{2}$, the error is so large that corrections to the $\mathrm{O}_{2}$ molecule became a standard way of addressing this issue for the RPBE [81] and BEEF-vdW [24] functional. If one assumes that the majority of the error in these reaction energies lies in the description of the gaseous molecules having double and triple bonds, an interesting question arises. How is the performance of the various functionals for surface reaction energies if we avoid use of gas-phase molecules with double and triple bonds? To do this, all adsorption energies for molecules with double or triple bonds in Table 2-4 were recalculated relative to the corresponding hydrogenated gas-phase molecule instead. For example, $\mathrm{H}_{2} \mathrm{O}$ has been used in place of $1 / 2 \mathrm{O}_{2 \text {,gas }}$ (minus $\mathrm{H}_{2, \text { gas }}$ ). Likewise, $\mathrm{CH}_{4}$ and $\mathrm{H}_{2} \mathrm{O}$ have been used instead of $\mathrm{CO}, \mathrm{NH}_{3}$ and $\mathrm{H}_{2} \mathrm{O}$ instead of $\mathrm{NO}$, and $2 \mathrm{NH}_{3}$ instead of $\mathrm{N}_{2}$. Cyclohexane has been used instead of benzene to avoid dealing with the aromatic system in gas phase. Thus, in Reaction 14 for example, which is $1 / 2 \mathrm{O}_{2}+\mathrm{Ni}(111) \rightarrow 0 / \mathrm{Ni}(111)$, DFT was used to calculate the energy for the alternate reaction $\mathrm{H}_{2} \mathrm{O}+\mathrm{Ni}(111) \rightarrow \mathrm{O} / \mathrm{Ni}(111)+\mathrm{H}_{2}$, and then that value was corrected by subtracting the experimental literature value for the reaction $\mathrm{H}_{2} \mathrm{O} \rightarrow \mathrm{H}_{2}+1 / 2 \mathrm{O}_{2}$ to arrive at the value tabulated here. This comparison is shown in Table 6 for a subset of Tables 2-4 containing CO, NO, $\mathrm{O}_{2}, \mathrm{~N}_{2}$, and benzene adsorption energies, where the errors of this alternate theoretical description are shown. 
Table 6. Errors in theoretical prediction of surface reaction energies when comparing to hydrogenated gas phase reactants to avoid DFT errors in gas molecules with double and triple bonds and aromaticity, in $\mathrm{kJ} / \mathrm{mol}$ as written.

\begin{tabular}{|c|c|c|c|c|c|c|c|c|}
\hline \multirow[b]{2}{*}{$\#$} & \multicolumn{2}{|c|}{$\begin{array}{l}\text { Reference corrected surface reaction energies } \\
\qquad(\mathrm{kJ} / \mathrm{mol} \text { as written })\end{array}$} & \multicolumn{6}{|c|}{ Errors (kJ/mol as written) } \\
\hline & Surface reaction & $\begin{array}{c}\Delta \mathrm{E}_{\text {exp }}-\Delta \mathrm{ZPE}- \\
\Delta \mathrm{E}_{\text {react }}\end{array}$ & LDA & PBEsol & PW91 & PBE & RPBE & BEEF-vdW \\
\hline 1 & $\mathrm{CO}+\mathrm{Ni}(111) \rightarrow \mathrm{CO} / \mathrm{Ni}(111)$ & 148 & -39 & -34 & -23 & -24 & -25 & -59 \\
\hline 2 & $\mathrm{CO}+\mathrm{Pt}(111) \rightarrow \mathrm{CO} / \mathrm{Pt}(111)$ & 148 & 16 & 4 & -2 & -4 & -20 & -46 \\
\hline 3 & $\mathrm{CO}+\mathrm{Pd}(111) \rightarrow \mathrm{CO} / \mathrm{Pd}(111)$ & 128 & -18 & -22 & -14 & -4 & -18 & -48 \\
\hline 4 & $\mathrm{CO}+\mathrm{Pd}(100) \rightarrow \mathrm{CO} / \mathrm{Pd}(100)$ & 115 & 7 & 3 & 6 & 20 & -6 & -30 \\
\hline 5 & $\mathrm{CO}+\mathrm{Rh}(111) \rightarrow \mathrm{CO} / \mathrm{Rh}(111)$ & 130 & 3 & -9 & -13 & -15 & -30 & -57 \\
\hline 6 & $\mathrm{CO}+\operatorname{Ir}(111) \rightarrow \mathrm{CO} / \operatorname{Ir}(111)$ & 108 & 25 & 11 & 4 & 2 & -14 & -43 \\
\hline 7 & $\mathrm{CO}+\mathrm{Cu}(111) \rightarrow \mathrm{CO} / \mathrm{Cu}(111)$ & 215 & 30 & 17 & 12 & 11 & -8 & -35 \\
\hline 8 & $\mathrm{CO}+\mathrm{Ru}(001) \rightarrow \mathrm{CO} / \mathrm{Ru}(001)$ & 111 & 26 & 12 & 7 & 6 & -10 & -38 \\
\hline 9 & $\mathrm{CO}+\mathrm{Co}(001) \rightarrow \mathrm{CO} / \mathrm{Co}(001)$ & 153 & -10 & -11 & -13 & -2 & -27 & -56 \\
\hline 10 & $\begin{array}{l}\mathrm{NO}+\mathrm{Ni}(100) \rightarrow \mathrm{N} / \mathrm{Ni}(100)+ \\
\mathrm{O} / \mathrm{Ni}(100)\end{array}$ & 138 & -199 & -161 & -132 & -108 & -116 & -129 \\
\hline 11 & $\mathrm{NO}+\mathrm{Pt}(111) \rightarrow \mathrm{NO} / \mathrm{Pt}(111)$ & 318 & -77 & -72 & -60 & -53 & -69 & -88 \\
\hline 12 & $\mathrm{NO}+\mathrm{Pd}(111) \rightarrow \mathrm{NO} / \mathrm{Pd}(111)$ & 255 & -53 & -48 & -37 & -26 & -46 & -62 \\
\hline 13 & $\mathrm{NO}+\mathrm{Pd}(100) \rightarrow \mathrm{NO} / \mathrm{Pd}(100)$ & 274 & -68 & -59 & -43 & -28 & -49 & -69 \\
\hline 14 & $\mathrm{O}_{2}+\mathrm{Ni}(111) \rightarrow 2 \mathrm{O} / \mathrm{Ni}(111)$ & 44 & -56 & -24 & -12 & -5 & 19 & -15 \\
\hline 15 & $\mathrm{O}_{2}+\mathrm{Ni}(100) \rightarrow 2 \mathrm{O} / \mathrm{Ni}(100)$ & -2 & -86 & -46 & -20 & 20 & 14 & -22 \\
\hline 16 & $\mathrm{O}_{2}+\mathrm{Pt}(111) \rightarrow 2 \mathrm{O} / \mathrm{Pt}(111)$ & 321 & -80 & -54 & -51 & -22 & -24 & -64 \\
\hline 17 & $\mathrm{O}_{2}+\mathrm{Rh}(100) \rightarrow 2 \mathrm{O} / \mathrm{Rh}(100)$ & 174 & -172 & -136 & -107 & -59 & -73 & -114 \\
\hline \multirow[t]{4}{*}{33} & $\begin{array}{l}\mathrm{C}_{6} \mathrm{H}_{6}+\mathrm{Pt}(111) \rightarrow \\
\mathrm{C}_{6} \mathrm{H}_{6} / \mathrm{Pt}(111)\end{array}$ & 123 & -26 & -3 & 54 & 52 & 86 & 12 \\
\hline & & Weighted MSE & -26.7 & -23.4 & -15.7 & -8.4 & -18.1 & -43.9 \\
\hline & & Weighted MAE & 38.6 & 28.6 & 24.9 & 19.7 & 29.5 & 45.3 \\
\hline & & Weighted RMSE & 47.2 & 37.9 & 32.5 & 26.2 & 37.3 & 49.9 \\
\hline
\end{tabular}

As can be seen in Table 6, the corrections have both a positive and negative influence on the description of surface reaction energies. Predictions of reaction energies involving $\mathrm{CO}$ for example improve significantly for LDA and PBEsol and also somewhat for PW91 and PBE. They worsen the description derived with the RPBE and BEEF-vdW functional, however. For the latter, a significant deviation from the experimental values is observed. This may not be surprising, as both RPBE and BEEF-vdW have been fitted to experimental values using uncorrected energies (i.e., relative to gas-phase molecules with double and triple bonds), so that a correction ought to make the predictions worse. A similar trend can be observed for the corrected reaction energies involving NO. For $\mathrm{O}_{2}$, however, an improvement is achieved even for RPBE and BEEF-vdW (see Table 6). Interestingly, for benzene, values improve significantly for all functionals if the reaction energies are taken relative to gas-phase cyclohexane and $\mathrm{H}_{2}$, with PBEsol and BEEF-vdW predicting values close to experiments. In general, large part of the behavior of the various 
functionals relate to a poor description of some gas-phase species. After correction schemes are used most of the GGAs perform better, while this improvement is not generally observed for BEEF-vdW as it was fitted to both gas-phase and adsorption energies data sets.

\section{CONCLUSIONS}

In summary, we have presented what we consider the most reliable collection of experimental reaction energies involving surface-adsorbate bonds chosen for benchmarking computations existing to date. The database consists of 39 reaction energies and exhibits 10 different transition metals as well as a large variety of adsorbates forming various metal- $\mathrm{N},-\mathrm{O},-\mathrm{C},-\mathrm{H},-\mathrm{I}$ bonds. We tested a selected set of DFT functionals against the experimentally obtained data and found that the performance of the functionals varies significantly. Both PW91 and PBE overestimate chemisorption energies for strong covalently bound adsorbates. When considering larger adsorbates with large van der Waals bonding contributions to the adsorption energy, the intrinsic overbinding of PW91 and PBE, which do not contain non-local correlation terms, compensate to some extent for the missing description of dispersion forces. RPBE performs very well for the strongly adsorbed systems, for which it was fitted, but fails considerably for species with large vdW contributions. Care has to be taken in the choice of comparison as PW91 and PBE functionals could be perceived as performing well on a mixed database of adsorption energies, where some adsorbates have large van der Waal's contributions, although their "perceived reasonable" performance stems from their systematic overbinding compensating for their lack of van der Waal's contributions. It should be stressed that even with this compensation neither PW91 nor PBE yields accurate results for vdW bonded systems. Overall, the BEEF-vdW seems to perform most satisfactorily among the selected functionals, in that it performs at the same level of accuracy as the RPBE functional for strong covalent chemisorption, while also performing quite reasonably for systems where $\mathrm{vdW}$ interactions are thought to contribute a large amount to the energy. It should be noted that there are other vdW-functionals that also yield satisfactorily results for vdW bonded systems [86,87]. The benchmarking of these functionals, however, is out of the scope of the parent paper. It is clear that large errors still persist for a variety of reactions considered here and that significant systematic improvements are desirable. For PW91 and PBE, improvements are possible with the correction of ill-described gas-phase molecules, while this is only partially true for RPBE and BEEF-vdW. This stems from the systematic error on the RPBE and BEEF-vdW functionals generally being smaller to begin with.

Finally, we suggest that new functionals aimed at the surface science community could be guided by the experimental benchmarks presented here. With the accessibility of accurate surface science experiments, however, it is expected that this database will expand significantly in the future, giving an even better foundation for calibrating electronic structure theories for surface science and catalysis applications. 


\section{ACKNOWLEDGEMENTS}

This work has been supported by the U.S. Department of Energy, Office of Science, Office of Basic Energy Sciences through grants to SUNCAT and through the Materials Genome Initiative, and by the National Science Foundation under SusChEM grant CHE1361939.

\section{REFERENCES}

[1] K. Burke, J. Chem. Phys. 136 (2012) 150901.

[2] A.J. Medford, J. Wellendorff, A. Vojvodic, F. Studt, F. Abild-Pedersen, K.W. Jacobsen, T. Bligaard, J.K. Nørskov, Science 345 (2014) 197-200.

[3] C.T. Campbell, J.R.V. Sellers, Chem. Rev. 113 (2013) 4106-4135.

[4] K. Christmann, Surf. Sci. Rep. 9 (1988) 1-163.

[5] J.B. Miller, H. R. Siddiqui, S.M. Gates, J.N. Russell, J.T. Yates, J.C. Tully, M.J. Cardillo, J.

Chem. Phys. 87 (1987) 6725-6732.

[6] C.T. Campbell, O. Lytken, Surf. Sci. 603 (2009) 1365-1372.

[7] W.A. Brown, R. Kose, D.A. King, Chem. Rev. 98 (1998) 797-831.

[8] B. Hammer, L.B. Hansen, J.K. Nørskov, Phys. Rev. B 59 (1999) 7413-7421.

[9] L.A. Curtiss, P.C. Redfern, K. Raghavachari, J. Chem. Phys. 126 (2007) 084108.

[10] J.P. Perdew, Y. Wang, Phys. Rev. B 45 (1992) 13244.

[11] J.P. Perdew, A. Ruzsinszky, G. Csonka, O. Vydrov, G. Scuseria, L. Constantin, X. Zhou, K. Burke, Phys. Rev. Lett. 100 (2008) 136406.

[12] J.P. Perdew, J. Chevary, S. Vosko, K. Jackson, M. Pederson, D. Singh, C. Fiolhais, Phys. Rev. B 46, (1992) 6671.

[13] J.P. Perdew, K. Burke, M. Ernzerhof, Phys. Rev. Lett. 77 (1996) 3865.

[14] J. Wellendorff, K.T. Lundgaard, A. Møgelhøj, V. Petzold, D.D. Landis, J.K. Nørskov, T.

Bligaard, K.W. Jacobsen, Phys. Rev. B 85 (2012) 235149.

[15] P. Giannozzi, S. Baroni, N. Bonini, M. Calandra, R. Car, C. Cavazzoni,

D. Ceresoli, G.L. Chiarotti, M. Cococcioni, et al, J. Phys.: Condens. Matter. 21 (2009) 395502.

[16] S.R. Bahn, K.W. Jacobsen, Comput. Sci. Eng. 4 (2002) 56.

[17] A.A. Adllan, A. Dal Corso, J. Phys.: Condens. Matter 23 (2011) 425501.

[18] K.F. Garrity, J.W. Bennett, K.M. Rabe, D. Vanderbilt, Comp. Mater. Sci. 81 (2014) 446.

[19] J. Nocedal, S. J. Wright, in: Numercial Optimization, Springer, New York, 2006.

[20] H.J. Monkhorst, J.D. Pack, Phys. Rev. B 13 (1976) 5188.

[21] J.S. Yoo, F. Abild-Pedersen, J.K. Nørskov, F. Studt, ACS Catal. 4 (2014) 1226.

[22] J.B. Miller, H.R. Siddiqui, S.M. Gates, J.N. Russell Jr., J.T. Yates Jr., J.C. Tully, M.J. Cardillo, J. Chem. Phys. 87 (1987) 6725.

[23] J.-H. Fischer-Wolfarth, J. Hartmann, J.A. Farmer, J.M. Flores-Camacho, C.T. Campbell, S. Schauermann, H.-J. Freund, Rev. Sci. Instrum. 82 (2011) 024102.

[24] E.M, Karp, C.T. Campbell, F. Studt, F. Abild-Pederson, J.K. Nørskov, H. Phys. Chem. C 116 (2012) 25772.

[25] C.T. Campbell, J.R.V. Sellers, J. Am. Chem. Soc. 123 (2012) 18109.

[26] W.D. Lew, M.C. Crowe, E. Karp, C.T. Campbell, J. Phys. Chem. C 115 (2011) 9164; and references therein

[27] W.D. Lew, M.C. Crowe, E. Karp, O. Lytken, J.A. Farmer, L. Arnadottir,C. Schoenbaum, C.T. Campbell, J. Phys. Chem. C 115 (2011) 11586; and references therein

[28] A.R. Bishop, G.S. Girolami, R.G. Nuzzo, J.Phys.Chem.B 104 (2000) 754.

[29] K. Christmann, O. Schober, G. Ertl, J. Chem. Phys. 60 (1974) 4719-4724.

[30] H. Froitzheim, U. Kohler, Surf. Sci. 188 (1987) 70-86.

[31] A. Schiesser, P. Hortz, R. Schafer, Surf. Sci. 604 (2010) 2098-2105.

[32] C.T. Campbell, G. Ertl, H. Kuipers, J. Segner, Surf. Sci. 107 (1981) 207-219.

[33] S.R. Kelemen, T.E. Fischer, J.A. Schwarz, Surf. Sci. 81 (1979) 440-450.

[34] B. Poelsema, R.L. Palmer, G.A. Comsa Surf. Sci. 136 (1984) 1-14

[35] I. Ratajczykowa, Surf. Sci. 172 (1986) 691-714.

[36] H. Conrad, G. Ertl, J. Koch, E.E. Latta, Surf. Sci. 43 (1974) 462-480. 
[37] T. Engel, G. Ertl, J. Chem. Phys. 69 (1978) 1267-1281.

[38] A. Noordermeer, G.A. Kok, B.E. Nieuwenhuys, Surf. Sci. 172 (1986) 349-362.

[39] G.A. Kok, A. Noordermeer, B.E. Nieuwenhuys, Surf. Sci. 135 (1983) 65-80.

[40] M.P. Kiskinova, G.M. Bliznakov, Surf. Sci. 123 (1982) 61-76.

[41] X.C. Guo, J.T. Yates, J. Chem. Phys. 90 (1989) 6761-6766.

[42] K. Christmann, G. Ertl, Surf. Sci. 33 (1972) 254.

[43] R.J. Behm, K. Christmann, G. Ertl, M.A. Vanhoven, J. Chem. Phys. 73 (1980) 2984-2995.

[44] J.C. Tracy, P.W. Palmberg, J. Chem. Phys. 51 (1969) 4852.

[45] J. Szanyi, D.W. Goodman, J. Phys. Chem. 98 (1994) 2972-2977.

[46] D.H Wei, D.C Skelton, S.D Kevan Surf. Sci. 381 (1997) 49-64.

[47] K.A. Peterlinz, T.J. Curtiss, S.J. Sibener, J. Chem. Phys. 95 (1991) 6972-6985.

[48] E.G. Seebauer, A.C.F. Kong, L.D. Schmidt, Appl. Surf. Sci. 31 (1988) 163-172.

[49] M. Sushchikh, J. Lauterbach, W.H. Weinberg, J. Vac. Sci. Technol., A 15 (1997) 1630-1634.

[50] B.J. Hinch, L.H. Dubois, Chem. Phys. Lett. 171 (1990) 131-135.

[51] P. Hollins, J. Pritchard, Surf. Sci. 89 (1979) 486-495.

[52] H. Pfnur, P. Feulner, H.A. Engelhardt, D. Menzel, Chem. Phys. Lett. 59 (1978) 481-486.

[53] J.C. Vickerman, K. Christmann, G. Ertl, J. Catal. 71 (1981) 175-191.

[54] J. Lahtinen, J. Vaari, K. Kauraala, Surf. Sci. 418 (1998) 502-510.

[55] V. Fiorin, D. Borthwick, D.A. King, Surf. Sci. 603 (2009) 1360-1364.

[56] R.D. Ramsier, Q. Gao, H.N. Waltenburg, K.W. Lee, O.W. Nooij, L. Lefferts, J.T. Yates, Surf.

Sci. 320 (1994) 209-237.

[57] Q.F. Ge, R. Kose, D.A. King, Adv. Catal. 45 (2000) 207-259.

[58] D.H. Parker, M.E. Bartram, B.E. Koel, Surf. Sci. 217 (1989) 489-510.

[59] C.T. Campbell, G. Ertl, H. Kuipers, J. Segner, Surf. Sci. 107 (1981) 220-236.

[60] F. Bozso, G. Ertl, M. Grunze, M. Weiss, J. Catal. 49 (1977) 18-41.

[61] B. Poelsema, K. Lenz, G. Comsa, J. Chem. Phys. 134 (2011) 074703.

[62] O. Lytken, W. Lew, J.J.W. Harris, E.K. Vestergaard, J.M. Gottfried, C.T. Campbell, J. Am.

Chem. Soc. 130 (2008) 10247-10257.

[63] K. Christmann, Surf. Sci. Rep. 9 (1988) 1-163.

[64] K. Christmann, Adsorbate properties of hydrogen on solid surfaces, in: Landolt-Börnstein, Group III, Vol. 42, Physics of Covered Solid Surfaces, Subvolume A, Part 5 (H.-P. Bonzel, ed.),

Springer, Berlin 2006

[65] J.T. Yates Jr., P.A. Thiel, W.H. Weinberg Surf. Sci. 84 (1979) 427.

[66] M. Labayen, S.A. Furman, D.A. Harrington, Surf. Sci. 525 (2003) 149-158.

[67] C.A. Wolcott, I.X. Green, T.L. Silbaugh, Y. Xu, C.T. Campbell, J. Phys. Chem. C 118 (2014) 29310-29321.

[68] E.M. Karp, T.L. Silbaugh, C.T. Campbell, J. Am. Chem. Soc. 135 (2013) 5208-5211.

[69] K.J. Wu, S.D. Kevan, J. Chem. Phys. 95 (1991) 5355-5363.

[70] E.M. Karp, T.L. Silbaugh, C.T. Campbell, J. Phys. Chem. C 117 (2013) 6325-6336.

[71] E.M. Karp, T.L. Silbaugh, M.C. Crowe, C.T. Campbell, J. Am. Chem. Soc. 134 (2012) 2038820395.

[72] S.L. Tait, Z. Dohnalek, C.T. Campbell, B.D. Kay, J. Chem. Phys. 125 (2006) 234308.

[73] H. Ihm, H.M. Ajo, J.M. Gottfried, P. Bera, C.T. Campbell, J. Phys. Chem. B 108 (2004) 14627-14633.

[74] M. Xi, M.X. Yang, S.K. Jo, B.E. Bent, P. Stevens, J. Chem. Phys. 101 (1994) 9122-9131.

[75] S. Lukas, S. Vollmer, G. Witte, C. Woll, J. Chem. Phys. 114 (2001) 10123-10130.

[76] X.L. Zhou, M.E. Castro, J.M. White, Surf. Sci. 238 (1990) 215-225.

[77] D. Syomin, J. Kim, B.E. Koel, G.B. Ellison, J. Phys. Chem. B 105 (2001) 8387-8394.

[78] Y.R. Luo, Comprehensive Handbook of Chemical Bond Energies, CRC Press, Boca Raton, FL, 2007

[79] W. Lew, M.C. Crowe, C.T. Campbell, J. Carrasco, A. Michaelides, J. Phys. Chem. C 115 (2011) 23008-23012.

[80] K. Lee, E.D. Murray, L. Kong, B.I. Lundqvist, and D.C. Langreth, Phys. Rev. B 82 (2010) 081101.

[81] J. Rossmeisl, A. Logadottir, J.K. Nørskov, Chem. Phys. 319 (2005) 178-184. 
[82] A.A. Peterson, F. Abild-Pedersen, F. Studt, J. Rossmeisl, J.K. Nørskov, Energy Environ. Sci. $3(2010) 1311$.

[83] F. Studt, F. Abild-Pedersen, J.B. Varley, J.K. Nørskov, Catal. Lett. 143 (2013) 71-73.

[84] R. Haunschild, W. Klopper, J. Chem. Phys. 136 (2012) 164102.

[85] L.A. Curtiss, K. Raghavachari, P.C. Redfern, J.A. Pople, J. Chem. Phys. 112 (2000) 73747383.

[86] J. Klimes, D.R. Bowler, A. Michaelides, J. Phys. Condens. Matter 22 (2010) 022201.

[87] J. Carraso, J. Klimes, A. Michaelides, J. Chem. Phys. 138 (2013) 024708. 